\title{
Correction to: Porcine Picornavirus 3C Protease Degrades PRDX6 to Impair PRDX6-mediated Antiviral Function
}

\author{
Congcong Wang ${ }^{1} \cdot$ Huanhuan Feng ${ }^{1} \cdot$ Xiangle Zhang $^{1} \cdot \mathrm{Kangli}^{\mathrm{Li}}{ }^{1} \cdot \mathrm{Fan} \mathrm{Yang}^{1} \cdot$ Weijun Cao $^{1} \cdot$ \\ Huisheng Liu $^{1} \cdot$ Lili Gao $^{1} \cdot$ Zhaoning Xue $^{1} \cdot$ Xiangtao Liu $^{1} \cdot$ Zixiang Zhu $^{1}$ (i) $\cdot$ Haixue Zheng ${ }^{1}$ (i)
}

Published online: 12 November 2021

(C) Wuhan Institute of Virology, CAS 2021

\section{Correction to: Virologica Sinica (2021) 36:948-957}

https://doi.org/10.1007/s12250-021-00352-4

Due to our negligence, the original version of this article, published online on March 15, 2021, contained a mistake in Figure 2E (The Knockdown band of Western blotting was provided incorrectly). The correct Fig. $2 \mathrm{E}$ is given below. We apologize for this error and state that this does not change the scientific conclusions of the article in any way.

Congcong Wang and Huanhuan Feng contributed equally to this work.

The original article can be found online at https:// doi.org/10.1007/s12250-021-00352-4.

Haixue Zheng

haixuezheng@163.com

$\triangle$ Zixiang Zhu

zhuzixiang@126.com

1 State Key Laboratory of Veterinary Etiological Biology, OIE/ National Foot and Mouth Diseases Reference Laboratory, Key Laboratory of Animal Virology of Ministry of Agriculture, Lanzhou Veterinary Research Institute, Chinese Academy of Agricultural Sciences, Lanzhou 730046, China 
A

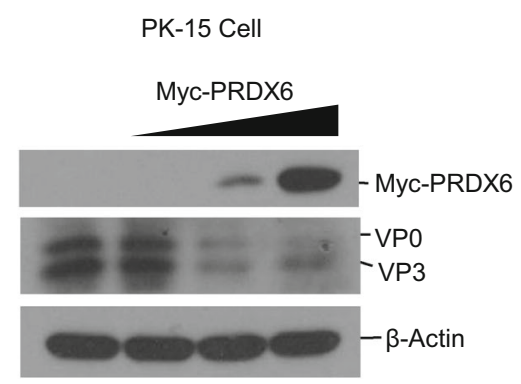

D

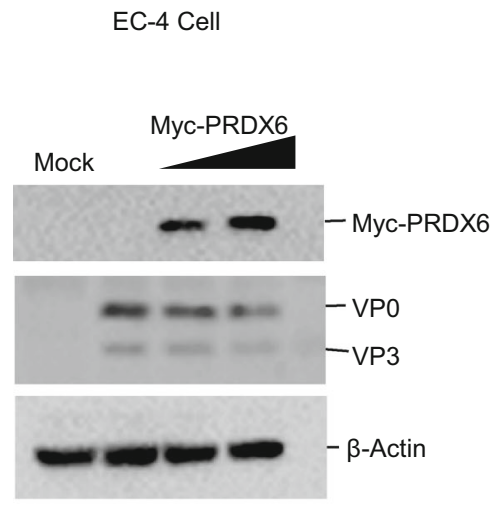

$\mathrm{F}$

PK-15 Cell

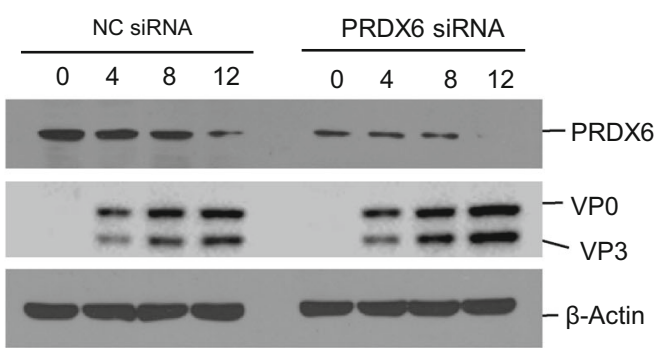

Fig. 2 PRDX6 inhibited FMDV replication. A-C PK-15 cells were transfected with $0,0.5,1$ or $2 \mu \mathrm{g}$ of Myc-PRDX6 expressing plasmids for $24 \mathrm{~h}$. The cells were then infected with FMDV (MOI $=0.5)$ for 12 h. The expression of viral proteins (A) and viral RNA (B) were detected by Western blotting and qPCR respectively. The virus yields were measured by $\mathrm{TCID}_{50}$ assay $(\mathbf{C})$. D EC- 4 cells were transfected with 0,1 or $2 \mu \mathrm{g}$ of Myc-PRDX6 expressing plasmids for $24 \mathrm{~h}$. The cells were then mock-infected or infected with FMDV for $12 \mathrm{~h}$. The expression of viral proteins and viral RNA were detected by Western blotting and qPCR respectively. E PK-15 cells were transfected with
C
PK-15 Cell

FMDV
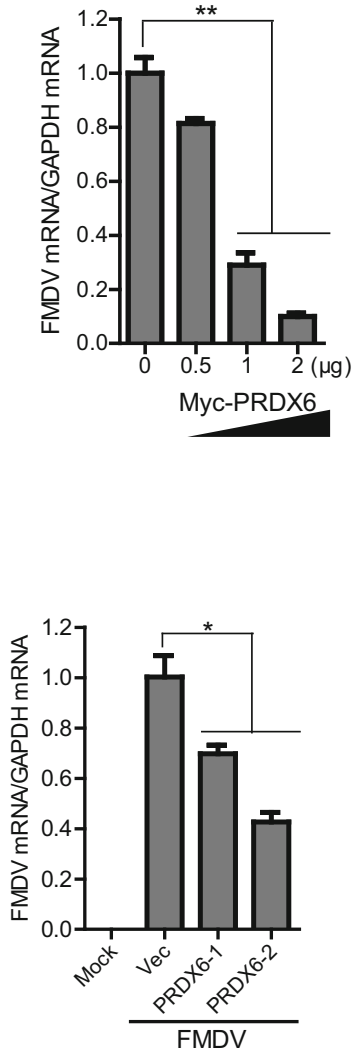

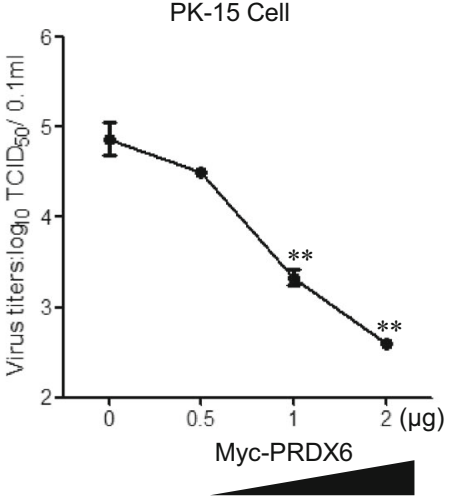

$E$

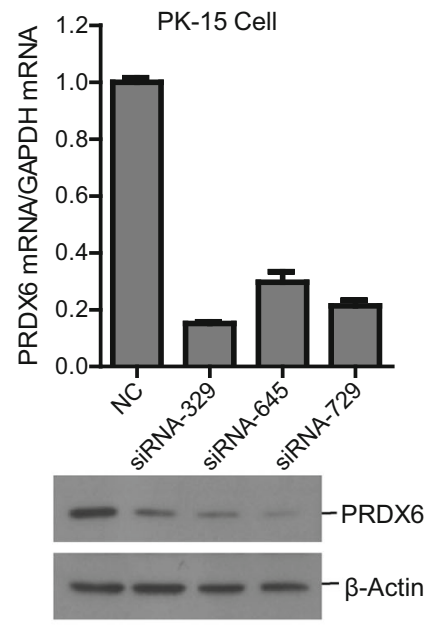

$\mathrm{H}$
G

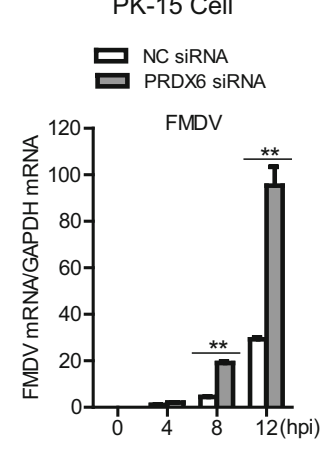

PK-15 Cell

$\rightarrow$ NC siRNA

¥ PRDX6 SiRNA

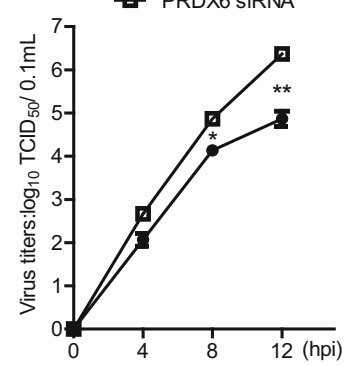

$120 \mathrm{nM}$ of nontargeting control NC siRNA or PRDX6 siRNA (siRNA-329, siRNA-645 siRNA-729) for $36 \mathrm{~h}$, the knockdown efficiency of each siRNA was then evaluated by qPCR and Western blotting analysis respectively. F-H PK-15 cells were transfected with $120 \mathrm{nM}$ of NC siRNA or PRDX6 siRNA (siRNA-329) for $36 \mathrm{~h}$, the cells were then infected with FMDV (MOI $=0.5$ ) for $0,4,8$ or $12 \mathrm{~h}$. The expression of viral proteins $(\mathbf{F})$ and viral RNA $(\mathbf{G})$ were detected by Western blotting and $\mathrm{qPCR}$ respectively. The virus yields were measured by $\mathrm{TCID}_{50}$ assay $(\mathbf{H})$. 